\title{
Existence of time homoclinic solutions for a class of discrete wave equations
}

Xinfu Li and Guang Zhang*

${ }^{*}$ Correspondence:

Ixyzhg@tjcu.edu.cn

School of Science, Tianjin University

of Commerce, Tianjin, 300134,

P.R. China

\section{Springer}

\begin{abstract}
In this paper, a class of nonperiodic discrete wave equations with Dirichlet boundary conditions are obtained by using the center-difference method. It is a strongly indefinite discrete Hamiltonian system. By using a variant and generalized weak linking theorem, the existence of the nontrivial time homoclinic solutions for the system will be obtained. The obtained main results here allow the classical Ambrosetti-Rabinowitz superlinear condition to be replaced by a general superquadratic condition. Such a method cannot be used for the corresponding continuous wave equations, however, it is valid for some general discrete Hamiltonian systems. Similarly, the existence of homoclinic periodic solutions can also be considered.
\end{abstract}

MSC: $39 \mathrm{~A} 14$

Keywords: discrete wave equation; difference system; homoclinic solutions; linking theorem; Ambrosetti-Rabinowitz condition; homoclinic periodic solution

\section{Introduction}

Consider a nonlinear wave equation of the form

$$
u_{t t}-u_{x x}+a u-\gamma(t) g(t, u)=0, \quad 0<x<\pi, t \in R,
$$

with the Dirichlet boundary conditions

$$
u(0, t)=0=u(\pi, t) .
$$

Let $Z$ be the set of all integers and $R$ be the set of all real numbers. For any integers $k$ and $l$ with $k<l$, denote $[k, l]=\{k, k+1, \ldots, l\}$. By using the center-difference method for the space variable $x$ and the time variable $t$, we can obtain a discrete analog of (1)-(2) of the form

$$
\left\{\begin{array}{l}
\frac{1}{h^{2}} \Delta^{2} u\left(\frac{i \pi}{N+1},(n-1) h\right)-\left(\frac{N+1}{\pi}\right)^{2} \nabla^{2} u\left(\frac{(i-1) \pi}{N+1}, n h\right)+a u\left(\frac{i \pi}{N+1}, n h\right) \\
\quad-\gamma(n h) g\left(n h, u\left(\frac{i \pi}{N+1}, n h\right)\right)=0, \quad i \in[1, N], n \in Z \\
u\left(\frac{0 \pi}{N+1}, n h\right)=u\left(\frac{(N+1) \pi}{N+1}, n h\right)=0, \quad n \in Z
\end{array}\right.
$$

where $h>0$ is the time step size, $N$ is a positive integer, and the space step size is $\pi /(N+1)$. Let

(c) $2015 \mathrm{Li}$ and Zhang. This article is distributed under the terms of the Creative Commons Attribution 4.0 International License (http://creativecommons.org/licenses/by/4.0/), which permits unrestricted use, distribution, and reproduction in any medium, provided you give appropriate credit to the original author(s) and the source, provide a link to the Creative Commons license, and indicate if changes were made. 


$$
u\left(\frac{i \pi}{N+1}, n h\right)=u_{n}^{i} \text { and } \quad \gamma(n h)=\gamma_{n}
$$

then we have

$$
\left\{\begin{array}{l}
\Delta^{2} u_{n-1}^{i}-\delta^{2} \nabla^{2} u_{n}^{i-1}+\alpha u_{n}^{i}-\gamma_{n} f\left(n, u_{n}^{i}\right)=0, \quad i \in[1, N], n \in Z, \\
u_{n}^{0}=0=u_{n}^{N+1}, \quad n \in Z
\end{array}\right.
$$

where

$$
\begin{aligned}
& \Delta^{2} u_{n-1}^{i}=u_{n+1}^{i}-2 u_{n}^{i}+u_{n-1}^{i}, \\
& \nabla^{2} u_{n}^{i-1}=u_{n}^{i+1}-2 u_{n}^{i}+u_{n}^{i-1}, \\
& \alpha=a h^{2}, \quad \delta^{2}=h^{2}\left(\frac{N+1}{\pi}\right)^{2},
\end{aligned}
$$

and

$$
f\left(n, u_{n}^{i}\right)=h^{2} g\left(n h, u\left(\frac{i \pi}{N+1}, n h\right)\right)
$$

Problem (4) can also be rewritten by vector and matrix in the form

$$
\Delta^{2} U_{n-1}+\delta^{2} A U_{n}+\alpha U_{n}-\gamma_{n} \nabla H\left(n, U_{n}\right)=0, \quad n \in Z,
$$

where

$$
\begin{aligned}
& U_{n}=\left(u_{n}^{1}, u_{n}^{2}, \ldots, u_{n}^{N}\right)^{T}, \quad H\left(n, U_{n}\right)=\sum_{i=1}^{N} \int_{0}^{u_{n}^{i}} f(n, s) d s \\
& A=\left(\begin{array}{ccccc}
2 & -1 & 0 & \cdots & 0 \\
-1 & 2 & -1 & & 0 \\
& \ldots & & \ldots & \\
0 & & -1 & 2 & -1 \\
0 & \ldots & 0 & -1 & 2
\end{array}\right)_{N \times N},
\end{aligned}
$$

and

$$
\nabla H\left(n, U_{n}\right)=\left(f\left(n, u_{n}^{1}\right), f\left(n, u_{n}^{2}\right), \ldots, f\left(n, u_{n}^{N}\right)\right)^{T}
$$

The aim of this paper is to study the existence of homoclinic solution for discrete wave equation (4), or equivalently (5). A homoclinic solution of (5) is a solution which is asymptotic to a constant state as the time variable $n \rightarrow \pm \infty$. In view of the Smale-Šil'nikovBirkhoff theorem, the dynamics near transversal homoclinic orbits is chaotic in the sense of Li-Yorke or Devaney; see [1-3] and the references therein. So, the research on the existence of homoclinic solutions plays an important role in the understanding of the complicated dynamical behavior. 
For discrete Hamiltonian systems similar to (5), thanks to the variational structure, the variational methods have contributed greatly to the investigation of the existence of homoclinic solutions, many solvability conditions are given. However, to the best of our knowledge, we find that among the related results one or more of the following conditions are needed:

(P1) The linear operator $L:\left(\Delta^{2}+\delta^{2} A+\alpha I_{N}\right)$ is positive definite, where $I_{N}$ is the $N \times N$ unit matrix; see [4] and [5].

(P2) The function $\gamma(t) H(t, U)$ is $T$-periodic in $t$, see [6-8] and [9].

(P3) In the superquadratic case, most papers need the Ambrosetti-Rabinowitz condition, that is, there exist constants $\mu>2$ and $M>0$ such that

$$
0<\mu H(t, U) \leq(\nabla H(t, U), U), \quad U \in R^{N},|U| \geq M, t \in[0, T]
$$

see [10] and [7].

In 2012, Chen and Ma [11] considered the following difference equation:

$$
L u_{n}-\omega u_{n}=\gamma_{n} f_{n}\left(u_{n}\right), \quad n \in Z, u_{n} \in R,
$$

where $L$ is a Jacobi operator given by $L u_{n}=a_{n} u_{n+1}+a_{n-1} u_{n-1}+b_{n} u_{n}$. Under the assumptions that $\gamma_{n}, f_{n}(u), a_{n}$, and $b_{n}$ are $T$-periodic sequences, they obtained the existence of homoclinic solution to (8) with Ambrosetti-Rabinowitz condition replaced by

$$
\left|f_{n}(u)\right| \leq b\left(1+|u|^{p-1}\right)
$$

where $b>0$ and $p>2$ are constants. In a recent paper, Lin and Zhou [5] considered (8) for the higher dimensional case $n \in Z^{m}$. By using the Mountain Pass lemma, they obtained the existence of homoclinic solution to (8) without periodic conditions on $\gamma_{n} f_{n}(u)$ and with Ambrosetti-Rabinowitz condition replaced by (9). However, they need the operator $L-\omega$ to be positive definite. For the general background of difference equations, one can refer to the monographs [12] and [13].

In this paper, we consider (5) for the case that $L=\Delta^{2}+\delta^{2} A+\alpha I_{N}$ is strongly indefinite, without periodic assumptions on $\gamma_{n}$ and $H(n, u)$, and with Ambrosetti-Rabinowitz condition replaced by (9). To the best of our knowledge, this is the first work on homoclinic solution without conditions (P1)-(P3). Without loss of generality, we assume that $f(n, 0)=0$ for $n \in Z$; then $\left\{U_{n}\right\}=\{0\}$ is a solution of (5), which is called the trivial solution. As usual, we say that a solution $U=\left\{U_{n}\right\}$ of (5) is homoclinic (to 0 ) if $\lim _{|n| \rightarrow \infty} U_{n}=0$. By using a variant generalized weak linking theorem, we prove the existence of nontrivial homoclinic solution of (5) emanating from 0.

For the continuous wave equation (1)-(2), to the best of our knowledge, there are not many results about the existence of homoclinic solution; see [14] for the existence of homoclinic traveling wave solution and [15] for the homoclinic solution.

This paper is organized as follows. In the next section, we give some preliminary results which will be used in the proof of the main results. The exact spectrum $\sigma(L)$ of the linear operator $L$ in $l^{2}$ (defined in Section 2) is given. In this case, we can easily give the conditions

$0 \notin \sigma(L), \quad \sigma(L) \cap(0, \infty) \neq \varnothing \quad$ and $\quad \sigma(L) \cap(-\infty, 0) \neq \varnothing$. 
Our approach is based on an application of a variant and generalized weak linking theorem for strongly indefinite problem developed by Schechter and Zou [16], also see Chen and Ma [11]. Thus, a variant generalized weak linking theorem is also given in this section. To obtain a homoclinic solution of (5), some compact condition is needed, so weighted sequence spaces $l_{\eta}^{p}$ are also introduced in this section. In Section 3, we first make some assumptions on $L$, and then we can give an orthogonal decomposition of $l^{2}$ according to $L$. At last, under some assumptions on $\gamma_{n}$ and $f(n, u)$, our main result is obtained by using the variant generalized weak linking theorem. Similarly, the existence of homoclinic periodic solutions for the discrete wave equation of the form

$$
\left\{\begin{array}{l}
\Delta^{2} u_{n-1}^{i}-\delta^{2} \nabla^{2} u_{n}^{i-1}+\alpha u_{n}^{i}-\gamma_{n} f\left(n, u_{n}^{i}\right)=0, \quad i, n \in Z, \\
\lim _{i \rightarrow \infty} u_{n}^{|i|}=0, \quad n \in Z
\end{array}\right.
$$

can also be considered.

As usual, $|\cdot|$ and $(\cdot, \cdot)$ denote the norm and the inner product in $R^{N}$, respectively. We use $c$ to represent positive constants which may change from line to line.

\section{Preliminaries}

In this section, we recall some basic facts which will be used in the proof of the main result.

\subsection{Function spaces}

Let

$$
X=\left\{U=\left\{U_{n}\right\}_{n \in Z}: U_{n} \in R^{N}, n \in Z\right\}
$$

and

$$
l^{p}=l^{p}(Z)=\left\{U \in X:\|U\|_{l^{p}}=\left(\sum_{n \in Z}\left|U_{n}\right|^{p}\right)^{1 / p}<\infty\right\} .
$$

Then the following embeddings between $l^{p}$ spaces hold (see [17]):

$$
l^{p} \subset l^{q}, \quad\|U\|_{l^{q}} \leq\|U\|_{l^{p}}, \quad 1 \leq p \leq q \leq \infty .
$$

For $p=2, l^{2}$ is a Hilbert space with the inner product

$$
\langle U, V\rangle_{l^{2}}=\sum_{n \in Z}\left(U_{n}, V_{n}\right), \quad U, V \in l^{2} .
$$

For a positive real valued bounded sequence $\eta=\left\{\eta_{n}: 0<\eta_{n} \leq \bar{\eta}<\infty\right\}_{n \in Z}$, we define the weighted sequence spaces $l_{\eta}^{p}$ :

$$
l_{\eta}^{p}=\left\{U \in X:\|U\|_{l_{\eta}^{p}}=\left(\sum_{n \in Z} \eta_{n}\left|U_{n}\right|^{p}\right)^{1 / p}<\infty\right\} .
$$

For a certain class of weight $\eta$, we have the following lemma (see [5]). 
Lemma 1 Assume that the positive real valued sequence $\eta=\left\{\eta_{n}\right\}_{n \in Z}$ satisfies $\lim _{|n| \rightarrow \infty} \eta_{n}=$ 0 . Then $l^{2} \hookrightarrow l_{\eta}^{2}$ with compact inclusion.

Define the functional $I$ on $l^{2}$ as follows:

$$
I(U)=\frac{1}{2} \sum_{n \in Z}\left(\Delta^{2} U_{n-1}, U_{n}\right)+\frac{1}{2} \delta^{2}\langle A U, U\rangle_{l^{2}}+\frac{1}{2} \alpha\|U\|_{l^{2}}^{2}-\sum_{n \in Z} \gamma_{n} H\left(n, U_{n}\right) .
$$

Then $I \in C^{1}\left(l^{2}, R\right)$ and, for any $U, V \in l^{2}$,

$$
\left\langle I^{\prime}(U), V\right\rangle_{l^{2}}=\sum_{n \in Z}\left(\Delta^{2} U_{n-1}+\delta^{2} A U_{n}+\alpha U_{n}-\gamma_{n} \nabla H\left(n, U_{n}\right), V_{n}\right),
$$

where $C^{1}\left(l^{2}, R\right)$ denotes the set of functionals that are Fréchet differentiable and their Fréchet derivatives are continuous on $l^{2}$.

Equation (14) implies that (5) is the Euler-Lagrange equation for $I$. Therefore, to find a nontrivial homoclinic solution of (5), one just needs to find a nonzero critical point of the functional $I$ on $l^{2}$.

\subsection{Linking theorem}

The abstract critical point theorem plays an important role in proving our main results. Let $E$ be a Hilbert space with norm $\|\cdot\|$ and inner product $\langle\cdot, \cdot\rangle$ and have an orthogonal decomposition $E=N \oplus N^{\perp}$, where $N \in E$ is a closed and separable subspace. Since $N$ is separable, we can define a new norm $|v|_{\omega}$ satisfying $|v|_{\omega} \leq\|v\|$ for all $v \in N$ and such that the topology induced by this norm is equivalent to the weak topology of $N$ on bounded subset of $N$. For $u=v+w \in E$ with $v \in N$ and $w \in N^{\perp}$, we define $|u|_{\omega}^{2}=|v|_{\omega}^{2}+\|w\|^{2}$, then $|u|_{\omega} \leq\|u\|$ for $u \in E$. Particularly, if $\left\{u_{n}=v_{n}+w_{n}\right\}_{n=1}^{\infty} \in E$ is $|\cdot|_{\omega}$-bounded and $u_{n} \rightarrow_{|\cdot|_{\omega}} u$, then $v_{n} \rightarrow v$ weakly in $N, w_{n} \rightarrow w$ strongly in $N^{\perp}, u_{n} \rightarrow v+w$ weakly in $E$ (see [16]).

Let $E=E^{-} \oplus E^{+}, z_{0} \in E^{+}$with $\left\|z_{0}\right\|=1$. For any $u \in E$, we write $u=u^{-} \oplus s z_{0} \oplus w^{+}$with $u^{-} \in E^{-}, s \in R, w^{+} \in\left(E^{-} \oplus R z_{0}\right)^{\perp}:=E_{1}^{+}$. For $R>0$, let

$$
Q=\left\{u=u^{-}+s z_{0} \mid s \in R^{+}, u^{-} \in E^{-},\|u\|<R\right\}
$$

with $p_{0}=s_{0} z_{0} \in Q, s_{0}>0$. We define

$$
D=\left\{u=s z_{0}+w^{+} \mid s \geq 0, w^{+} \in E_{1}^{+},\left\|s z_{0}+w^{+}\right\|=s_{0}\right\} .
$$

For $I \in C^{1}(E, R)$, define $h:[0,1] \times \bar{Q} \rightarrow E$ is $|\cdot|_{\omega}$-continuous, $h(0, u)=u, I(h(s, u)) \leq I(u)$ for $u \in \bar{Q}$, for any $\left(s_{0}, u_{0}\right) \in[0,1] \times \bar{Q}$, there is a $|\cdot|_{\omega}$-neighborhood $U_{\left(s_{0}, u_{0}\right)}$ such that

$$
\left\{u-h(t, u) \mid(t, u) \in U_{\left(s_{0}, u_{0}\right)} \cap[0,1] \times \bar{Q}\right\} \subset E_{\text {fin }},
$$

where $E_{\text {fin }}$ denotes various finite-dimensional subspaces of $E$ whose exact dimension are irrelevant and depend on $\left(s_{0}, u_{0}\right)$. Denote

$$
\Gamma=\{h \mid h:[0,1] \times \bar{Q} \rightarrow E\},
$$

then $\Gamma \neq \varnothing$ since $i d \in \Gamma$. 
The variant weak linking theorem is the following.

Lemma 2 (see [16]) The family of $C^{1}$-functional $\left\{I_{\lambda}\right\}$ has the form

$$
I_{\lambda}(u)=J(u)-\lambda K(u) \quad \text { for } \lambda \in[1,2] .
$$

\section{Assume that}

(a) $K(u) \geq 0, u \in E, I_{1}=I$

(b) $J(u) \rightarrow \infty$ or $K(u) \rightarrow \infty$ as $\|u\| \rightarrow \infty$;

(c) $I_{\lambda}$ is $|\cdot|_{\omega}$-upper semicontinuous, $I_{\lambda}^{\prime}$ is weakly sequentially continuous on $E$ moreover, $I_{\lambda}$ maps bounded sets to bounded sets;

(d) $\sup _{\partial Q} I_{\lambda} \leq \inf _{D} I_{\lambda}$ for $\lambda \in[1,2]$.

Then for almost all $\lambda \in[1,2]$, there exists a sequence $\left\{u_{n}\right\}$ such that

$$
\sup _{n}\left\|u_{n}\right\|<\infty, \quad I_{\lambda}^{\prime}\left(u_{n}\right) \rightarrow 0, \quad I_{\lambda}\left(u_{n}\right) \rightarrow c_{\lambda},
$$

where

$$
c_{\lambda}=\inf _{h \in \Gamma} \sup _{u \in Q} I_{\lambda}(h(1, u)) \in\left[\inf _{D} I_{\lambda}, \sup _{\bar{Q}} I\right] .
$$

\subsection{Spectrum results}

In the following, we give some results as regards the spectrum of operator $L=\Delta^{2}+\delta^{2} A+$ $\alpha I_{N}$ in $l^{2}$, where $L: l^{2} \rightarrow l^{2}$ is defined as $L U=\Delta^{2} U_{n-1}+\delta^{2} A U_{n}+\alpha U_{n}$ for $n \in Z$ and $U \in l^{2}$.

It is well known that the eigenvalues of $A$ are

$$
\eta_{k}=4 \sin ^{2} \frac{k \pi}{2(N+1)}, \quad k=1,2, \ldots, N
$$

and there is no other spectrum for $A$; see [13]. For any $U$ and $V \in l^{2}$, we have

$$
\left\|-\Delta^{2} U\right\|_{l^{2}} \leq 4\|U\|_{l^{2}}, \quad \sum_{n \in Z}\left(-\Delta^{2} U_{n-1}, V_{n}\right)=\sum_{n \in Z}\left(\Delta U_{n}, \Delta V_{n}\right)
$$

that is, $-\Delta^{2}$ is a bounded self-adjoint operator on $l^{2}$, and the spectrum $\sigma\left(-\Delta^{2}\right)$ is contained in $[0,4]$. Further, $\sigma\left(-\Delta^{2}\right)=[0,4]$; see [17]. By direct calculation, it is not hard to see that $-\Delta^{2}$ does not have eigenvalues in $l^{2}$. Therefore, the spectrum of $L$ is $\sigma(L)=\bigcup_{k=1}^{N}\{[-4,0]+$ $\left.\delta^{2} \eta_{k}+\alpha\right\}$.

\section{Main results}

In this paper, we assume that 0 lies in a gap of $\sigma(L)$, that is,

(S0) $\sup (\sigma(L) \cap(-\infty, 0))<0<\inf (\sigma(L) \cap(0, \infty))$, where $L=\Delta^{2}+\delta^{2} A+\alpha I_{N}$ is defined in Section 2 .

Note that assumption (S0) is satisfied if

$$
\delta^{2}>1 /\left(\sin ^{2} \frac{2 \pi}{2(N+1)}-\sin ^{2} \frac{\pi}{2(N+1)}\right)
$$


and

$$
\alpha \in\left(-4 \delta^{2} \sin ^{2} \frac{2 \pi}{2(N+1)}+4,-4 \delta^{2} \sin ^{2} \frac{\pi}{2(N+1)}\right) .
$$

Indeed, if this is the case, we see that the two spectrum intervals $S_{1}=\left\{[-4,0]+\delta^{2} \eta_{1}+\alpha\right\}$ and $S_{2}=\left\{[-4,0]+\delta^{2} \eta_{2}+\alpha\right\}$ are disjoint, $S_{1} \subset(-\infty, 0)$ and $S_{2} \subset(0, \infty)$.

Noting that $L$ is a bounded self-adjoint operator, and by assumption (S0), we have the following assertion (see [18]).

There exist a closed subspace $N$ of $l^{2}$ and a constant $\beta>0$ such that

(i) $L(N) \subset N$;

(ii) $\langle L U, U\rangle_{l^{2}} \geq \beta\|U\|_{l^{2}}^{2}, \forall U \in N$;

(iii) $\langle L U, U\rangle_{l^{2}} \leq-\beta\|U\|_{l^{2}}^{2}, \forall U \in N^{\perp}$.

Setting $E^{-}=N^{\perp}$ and $E^{+}=N$, it follows that $L\left(E^{+}\right)=E^{+}, L\left(E^{-}\right)=E^{-}$and that $l^{2}=E^{+} \oplus E^{-}$. Throughout this paper, for any $U \in l^{2}$, we always denote by $U^{+}$and $U^{-}$the vectors in $l^{2}$ with $U=U^{+}+U^{-}, U^{+} \in E^{+}$and $U^{-} \in E^{-}$. Evidently,

$$
\pm\left\langle L U^{ \pm}, U^{ \pm}\right\rangle_{l^{2}} \geq \beta\left\|U^{ \pm}\right\|_{l^{2}}^{2}, \quad \forall U^{ \pm} \in E^{ \pm}
$$

Therefore, we have

$$
\left\langle L\left(U^{+}+U^{-}\right), U^{+}-U^{-}\right\rangle_{l^{2}} \geq \beta\left\|U^{+}+U^{-}\right\|_{l^{2}}^{2}
$$

which together with $\left\|U^{+}+U^{-}\right\|_{l^{2}}=\left\|U^{+}-U^{-}\right\|_{l^{2}}$ implies

$$
\left\|L\left(U^{+}+U^{-}\right)\right\|_{l^{2}} \geq \beta\left\|U^{+}+U^{-}\right\|_{l^{2}} .
$$

For all $U, V \in l^{2}, U=U^{+}+U^{-}$and $V=V^{+}+V^{-}$, we can define an equivalent inner product $\langle\cdot, \cdot\rangle$ and the corresponding norm $\|\cdot\|$ in $l^{2}$ by

$$
\langle U, V\rangle=\left\langle L U^{+}, V^{+}\right\rangle_{l^{2}}-\left\langle L U^{-}, V^{-}\right\rangle_{l^{2}} \quad \text { and } \quad\|U\|=\langle U, U\rangle^{1 / 2},
$$

respectively. Therefore, $I$ defined in (13) can be rewritten as

$$
I(U)=\frac{1}{2}\left(\left\|U^{+}\right\|^{2}-\left\|U^{-}\right\|^{2}\right)-\sum_{n \in Z} \gamma_{n} H\left(n, U_{n}\right)
$$

In order to apply Lemma 2, we consider the family of functional defined by

$$
I_{\lambda}(U)=\frac{1}{2}\left\|U^{+}\right\|^{2}-\lambda\left(\frac{1}{2}\left\|U^{-}\right\|^{2}+\sum_{n \in Z} \gamma_{n} H\left(n, U_{n}\right)\right) .
$$

Then $I_{\lambda} \in C^{1}\left(l^{2}, R\right)$ and for any $U, V \in l^{2}$,

$$
\left\langle I_{\lambda}^{\prime}(U), V\right\rangle=\left\langle U^{+}, V^{+}\right\rangle-\lambda\left(\left\langle U^{-}, V^{-}\right\rangle+\sum_{n \in Z} \gamma_{n}\left(\nabla H\left(n, U_{n}\right), V_{n}\right)\right) .
$$

To continue the discussion, we make the following assumptions: 
(A1) $f(n, u)$ is continuous in $u, f(n, u)=o(|u|)$ as $|u| \rightarrow 0$ uniformly for $n \in Z$;

(A2) there exist constants $b_{1}>0$ and $p>2$ such that

$$
|f(n, u)| \leq b_{1}\left(1+|u|^{p-1}\right)
$$

uniformly for $n \in Z$ and $u \in R$;

(A3) $F(n, u) \geq 0$ for $n \in Z$ and $u \in R$, and

$$
\lim _{|u| \rightarrow \infty}\left(F(n, u) /|u|^{2}\right)=\infty
$$

uniformly for $n \in Z$, where $F(n, u)=\int_{0}^{u} f(n, s) d s$;

(A4) there exist constants $b_{2}>0$ and $q \in[p-1,2(p-1)]$ such that

$$
\frac{1}{2} f(n, u) u-F(n, u) \geq b_{2}|u|^{q}
$$

uniformly for $n \in Z$;

(A5) the positive real valued sequence $\gamma=\left\{\gamma_{n}\right\}_{n \in Z}$ satisfies $\sum_{n \in Z} \gamma_{n}^{2}<\infty$.

Note that our conditions are different from [11] and [5]. As is shown in the following examples, our assumptions are reasonable and there are cases in which the well-known Ambrosetti-Rabinowitz superquadratic condition (7) is not satisfied.

\section{Example Let}

$$
f_{1}(n, u)=u \ln (1+|u|)
$$

and

$$
F_{2}(n, u)=|u|^{p}+(p-2)|u|^{p-\epsilon} \sin ^{2}\left(|u|^{\epsilon} / \epsilon\right)+|u|^{q}
$$

where $p>2,0<\epsilon<p-2$, and $q \in(\max \{2, p-1\}, p]$. It is not hard to check that $f_{1}(n, u)$ and $F_{2}(n, u)$ satisfy (A1)-(A4) but do not satisfy the Ambrosetti-Rabinowitz superquadratic condition.

The condition (A5) is easy, for example, we consider that the sequence

$$
\gamma_{n}= \begin{cases}\frac{1}{n^{\alpha}}, & n \neq 0 \\ 1, & n=0\end{cases}
$$

which satisfies the condition $\sum_{n \in Z} \gamma_{n}^{2}<\infty$ when $\alpha>1$.

Under conditions (A3) and (A5), it is easy to see that $I_{\lambda}$ satisfies conditions (a) and (b) in Lemma 2. To see (c), we have the following lemma.

Lemma 3 Assume that (S0) and (A1)-(A5) hold, then $I_{\lambda}$ satisfies condition (c) in Lemma 2 for any $\lambda \in[1,2]$.

Proof It is easy to see that $I_{\lambda}$ maps bounded sets to bounded sets. 
Noting that $U^{(k)}=U^{(k)+}+U^{(k)-} \stackrel{|\cdot|_{\omega}}{\rightarrow} U=U^{+}+U^{-}$implies that $U^{(k)+} \rightarrow U^{+}$strongly in $l^{2}$, $U^{(k)-} \rightarrow U^{-}$weakly in $l^{2}$ and $U^{(k)} \rightarrow U$ weakly in $l^{2}$. Therefore, $\liminf _{k \rightarrow \infty}\left\|U^{(k)-}\right\|^{2} \geq$ $\left\|U^{-}\right\|^{2}$ and $U_{n}^{(k)} \rightarrow U_{n}$ for any $n \in Z$. Using Fatou's lemma, we know

$$
\liminf _{k \rightarrow \infty} \sum_{n \in Z} \gamma_{n} H\left(n, U_{n}^{(k)}\right) \geq \sum_{n \in Z} \liminf _{k \rightarrow \infty} \gamma_{n} H\left(n, U_{n}^{(k)}\right)=\sum_{n \in Z} \gamma_{n} H\left(n, U_{n}\right)
$$

Therefore, $\lim \sup _{k \rightarrow \infty} I_{\lambda}\left(U^{(k)}\right) \leq I_{\lambda}(U)$. That is, $I_{\lambda}$ is $|\cdot|_{\omega}$-upper semicontinuous.

Let $U^{(k)} \rightarrow U$ weakly in $l^{2}$, then $U_{n}^{(k)} \rightarrow U_{n}$ for any $n \in Z$ and $\left\{U^{(k)}\right\}$ is bounded in $l^{2}$. Thus, for any $W \in l^{2}$ with compact support, we have

$$
\sum_{n \in Z} \gamma_{n}\left(\nabla H\left(n, U_{n}^{(k)}\right), W_{n}\right) \rightarrow \sum_{n \in Z} \gamma_{n}\left(\nabla H\left(n, U_{n}\right), W_{n}\right), \quad k \rightarrow \infty
$$

Taking into account that the sequence $\left\{\gamma_{n} \nabla H\left(n, U_{n}^{(k)}\right)\right\}_{n \in Z}$ is bounded in $l^{2}$, we may replace $W$ by $V \in l^{2}$. Therefore,

$$
\left\langle I_{\lambda}^{\prime}\left(U^{(k)}\right), V\right\rangle \rightarrow\left\langle I_{\lambda}^{\prime}(U), V\right\rangle, \quad \forall V \in l^{2}
$$

That is, $I_{\lambda}^{\prime}$ is weakly sequentially continuous on $l^{2}$.

To continue the discussion, we still need to verify condition (d) in Lemma 2. Indeed, we have (see [11]) the following.

Lemma 4 Assume that (S0) and (A1)-(A5) hold, then one has the following:

(i) There exists $\rho>0$ independent of $\lambda \in[1,2]$ such that

$$
\kappa_{\lambda}:=\inf I_{\lambda}\left(B_{\rho} E^{+}\right)>0
$$

where $B_{\rho} E^{+}:=\left\{U \in E^{+}:\|U\|=\rho\right\}$. Moreover, $\kappa=\inf _{\lambda \in[1,2]} \kappa_{\lambda}>0$.

(ii) For fixed $\bar{W} \in E^{+}$with $\|\bar{W}\|=1$ and any $\lambda \in[1,2]$, there exists $R>\rho$ such that $\sup I_{\lambda}(\partial Q) \leq 0$, where $Q:=\left\{U:=V+s \bar{W}: s \geq 0, V \in E^{-},\|U\|<R\right\}$.

Proof (i) By conditions (A1) and (A2), we know that for any $\epsilon>0$ there exists $c_{\epsilon}>0$ such that

$$
|f(n, u)| \leq \epsilon|u|+c_{\epsilon}|u|^{p-1}, \quad n \in Z, u \in R
$$

which together with (A4) implies

$$
|F(n, u)| \leq \epsilon|u|^{2}+c_{\epsilon}|u|^{p} .
$$

Consequently,

$$
|H(n, V)| \leq \epsilon|V|^{2}+c_{\epsilon}|V|^{p}, \quad n \in Z, V \in R^{N},
$$


where $p>2$ is the parameter in (A2). By (12) and (18), for any $U \in E^{+}$and $\lambda \in[1,2]$, we have

$$
\begin{aligned}
I_{\lambda}(U) & \geq \frac{1}{2}\|U\|^{2}-2 \bar{\gamma} \sum_{n \in Z}\left(\epsilon\left|U_{n}\right|^{2}+c_{\epsilon}\left|U_{n}\right|^{p}\right) \\
& \geq \frac{1}{2}\|U\|^{2}-c \epsilon\|U\|^{2}-c_{\epsilon}\|U\|^{p},
\end{aligned}
$$

where $\bar{\gamma}=\sup _{n \in Z} \gamma_{n}$, and $c>0$ is a constant independently with $\epsilon, U$, and $\lambda$. The above inequality implies the conclusion.

(ii) Suppose by contradiction that there exists $U^{(k)} \in E^{-} \oplus R^{+} \bar{W}$ such that $I_{\lambda}\left(U^{(k)}\right)>0$ for all $k$ and $\left\|U^{(k)}\right\| \rightarrow \infty$ as $k \rightarrow \infty$. Set

$$
W^{(k)}=U^{(k)} /\left\|U^{(k)}\right\|=s_{k} \bar{W}+W^{(k)-} .
$$

By (A3) and (A5), we have

$$
\begin{aligned}
0<\frac{I_{\lambda}\left(U^{(k)}\right)}{\left\|U^{(k)}\right\|^{2}} & =\frac{1}{2}\left(s_{k}^{2}-\lambda\left\|W^{(k)-}\right\|^{2}\right)-\lambda \sum_{n \in Z} \gamma_{n} \frac{H\left(n, U_{n}^{(k)}\right)}{\left\|U^{(k)}\right\|^{2}} \\
& \leq \frac{1}{2}\left(s_{k}^{2}-\lambda\left\|W^{(k)-}\right\|^{2}\right) .
\end{aligned}
$$

Therefore,

$$
\left\|W^{(k)-}\right\|^{2} \leq \lambda\left\|W^{(k)-}\right\|^{2}<s_{k}^{2}=1-\left\|W^{(k)-}\right\|^{2} .
$$

It follows that $\left\|W^{(k)-}\right\| \leq \frac{1}{\sqrt{2}}$ and $\frac{1}{\sqrt{2}} \leq s_{k} \leq 1$.

Going to a subsequence if necessary, we have $s_{k} \rightarrow s \in\left[\frac{1}{\sqrt{2}}, 1\right], W^{(k)} \rightarrow W$ weakly in $l^{2}$ and $W_{n}^{(k)} \rightarrow W_{n}$ for any $n \in Z$ as $k \rightarrow \infty$. Hence $W=s \bar{W}+W^{-} \neq 0$. Then there exist $n_{0} \in Z$ and $j \in[1, N]$ such that $W_{n_{0}}^{j} \neq 0$, where $W_{n_{0}}^{j}$ denotes the $j$ th component of $W_{n_{0}}$. It follows that

$$
U_{n_{0}}^{(k) j}=W_{n_{0}}^{(k) j}\left\|U^{(k)}\right\| \rightarrow \infty, \quad \text { as } k \rightarrow \infty
$$

Combining (A3) and $\gamma_{n}>0$, we have

$$
\sum_{n \in Z} \gamma_{n} \frac{H\left(n, U_{n}^{(k)}\right)}{\left\|U^{(k)}\right\|^{2}} \geq \gamma_{n_{0}} \frac{F\left(n_{0}, U_{n_{0}}^{(k) j}\right)}{\left(U_{n_{0}}^{(k) j}\right)^{2}} \frac{\left(U_{n_{0}}^{(k) j}\right)^{2}}{\left\|U^{(k)}\right\|^{2}} \rightarrow \infty,
$$

as $k \rightarrow \infty$, which contradicts (19). The proof is completed.

Applying Lemmas 2, 3, and 4, we immediately obtain the following facts.

Lemma 5 Under assumptions (S0) and (A1)-(A5), for almost all $\lambda \in[1,2]$, there exists a sequence $\left\{U^{(k)}\right\}$ such that

$$
\sup _{k}\left\|U^{(k)}\right\|<\infty, \quad I_{\lambda}^{\prime}\left(U^{(k)}\right) \rightarrow 0, \quad I_{\lambda}\left(U^{(k)}\right) \rightarrow c_{\lambda} \in\left[\kappa, \sup _{\bar{Q}} I\right]
$$

where $Q$ and $\kappa$ are defined in Lemma 4. 
Lemma 6 Under the assumptions of Lemma 5 , for almost all $\lambda \in[1,2]$, there exists $U_{\lambda}$ with $U_{\lambda}^{+} \neq 0$ such that

$$
I_{\lambda}^{\prime}\left(U_{\lambda}\right)=0, \quad I_{\lambda}\left(U_{\lambda}\right) \leq \sup _{\bar{Q}} I
$$

Proof Let $\left\{U^{(k)}=U^{(k)+}+U^{(k)-}\right\}$ be the sequence obtained in Lemma 5 . Then there exists a subsequence, still denoted by the same notation, such that $U^{(k)} \rightarrow U, U^{(k)+} \rightarrow U^{+}$in $l^{2}$, $U_{n}^{(k)} \rightarrow U_{n}$ for any $n \in Z$ and

$$
U^{(k)+} \rightarrow U^{+} \quad \text { in } l_{\gamma}^{2}
$$

Step 1 . We show that $U^{(k)+} \rightarrow U^{+}$in $l^{2}$. By direct calculation, we have

$$
\begin{aligned}
\left\|U^{(k)+}-U^{+}\right\|^{2}= & \left\langle I_{\lambda}^{\prime}\left(U^{(k)}\right)-I_{\lambda}^{\prime}(U), U^{(k)+}-U^{+}\right\rangle \\
& +\lambda \sum_{n \in Z} \gamma_{n}\left(\nabla H\left(n, U_{n}^{(k)}\right)-\nabla H\left(n, U_{n}\right), U_{n}^{(k)+}-U_{n}^{+}\right) .
\end{aligned}
$$

By $U^{(k)+} \rightarrow U^{+}$in $l^{2}$, we have $\left\langle I_{\lambda}^{\prime}(U), U^{(k)+}-U^{+}\right\rangle \rightarrow 0$; and by the Hölder inequality, we obtain

$$
\begin{aligned}
\left\langle I_{\lambda}^{\prime}\left(U^{(k)}\right), U^{(k)}-U\right\rangle & \leq\left\|I_{\lambda}^{\prime}\left(U^{(k)}\right)\right\|\left\|U^{(k)}-U\right\| \\
& \leq \frac{1}{2}\left\|U^{(k)}-U\right\|^{2}+\frac{1}{2}\left\|I_{\lambda}^{\prime}\left(U^{(k)}\right)\right\|^{2} .
\end{aligned}
$$

By (12), (17), (21), and the Hölder inequality, we have

$$
\begin{aligned}
& \sum_{n \in Z} \gamma_{n}\left(\nabla H\left(n, U_{n}^{(k)}\right)-\nabla H\left(n, U_{n}\right), U_{n}^{(k)+}-U_{n}^{+}\right) \\
& \quad \leq c \sum_{n \in Z} \gamma_{n}\left(\left|U_{n}^{(k)}\right|+\left|U_{n}\right|+\left|U_{n}^{(k)}\right|^{p-1}+\left|U_{n}\right|^{p-1}\right)\left|U_{n}^{(k)+}-U_{n}^{+}\right| \\
& \quad \leq c\left(\left\|U^{(k)}\right\|_{l^{2}}+\|U\|_{l^{2}}+\left\|U^{(k)}\right\|_{l^{2(p-1)}}^{p-1}+\|U\|_{l^{2(p-1)}}^{p-1}\right)\left\|U^{(k)+}-U^{+}\right\|_{l_{\gamma}^{2}} \\
& \quad \leq c\left(\left\|U^{(k)}\right\|_{l^{2}}+\|U\|_{l^{2}}+\left\|U^{(k)}\right\|_{l^{2}}^{p-1}+\|U\|_{l^{2}}^{p-1}\right)\left\|U^{(k)+}-U^{+}\right\|_{l_{\gamma}^{2}} \rightarrow 0
\end{aligned}
$$

as $k \rightarrow \infty$. Inserting the above arguments into (22), we obtain

$$
\lim _{j \rightarrow \infty}\left\|U^{(k)+}-U^{+}\right\|=0
$$

Step 2. We claim that there exists a constant $\theta>0$ such that

$$
\lim _{k \rightarrow \infty}\left\|U^{(k)+}\right\| \geq \theta
$$

Indeed, if not, then $\lim _{k \rightarrow \infty}\left\|U^{(k)+}\right\|=0$. Therefore,

$$
2 I_{\lambda}\left(U^{(k)}\right) \leq\left\|U^{(k)+}\right\|^{2} \rightarrow 0
$$

as $k \rightarrow \infty$, which contradicts with the fact that $I_{\lambda}\left(U^{(k)}\right) \geq \kappa>0$. Hence (23) holds. 
By Step 1 and 2 , we obtain $U^{+} \neq 0$. Consequently, $U \neq 0$. It is obvious that

$$
\left\langle I_{\lambda}^{\prime}(U), V\right\rangle=\lim _{k \rightarrow \infty}\left\langle I_{\lambda}^{\prime}\left(U^{(k)}\right), V\right\rangle=0, \quad \forall V \in l^{2}
$$

Applying (A4) and Fatou's lemma, we have

$$
\begin{aligned}
\sup _{\bar{Q}} I \geq c_{\lambda} & =\lim _{k \rightarrow \infty}\left(I_{\lambda}\left(U^{(k)}\right)-\frac{1}{2}\left\langle I_{\lambda}^{\prime}\left(U^{(k)}\right), U^{(k)}\right\rangle\right) \\
& =\lim _{k \rightarrow \infty} \lambda \sum_{n \in Z} \gamma_{n}\left(\frac{1}{2}\left(\nabla H\left(n, U_{n}^{(k)}\right), U_{n}^{(k)}\right)-H\left(n, U_{n}^{(k)}\right)\right) \\
& \geq \lambda \sum_{n \in Z} \gamma_{n}\left(\frac{1}{2}\left(\nabla H\left(n, U_{n}\right), U_{n}\right)-H\left(n, U_{n}\right)\right)=I_{\lambda}(U) .
\end{aligned}
$$

Setting $U_{\lambda}=U$, we complete the proof.

Lemma 7 Under the assumptions of Lemma 5 , there exist $\lambda_{k} \rightarrow 1$ and a sequence $\left\{U^{\left(\lambda_{k}\right)}\right\}$ with $U^{\left(\lambda_{k}\right)+} \neq 0$ such that

$$
I_{\lambda_{k}}^{\prime}\left(U^{\left(\lambda_{k}\right)}\right)=0, \quad I_{\lambda_{k}}\left(U^{\left(\lambda_{k}\right)}\right) \leq \sup _{\bar{Q}} I
$$

Moreover, $\left\{U^{\left(\lambda_{k}\right)}\right\}$ is bounded.

Proof The existence of $\left\{U^{\left(\lambda_{k}\right)}\right\}$ with $U^{\left(\lambda_{k}\right)+} \neq 0$ such that

$$
I_{\lambda_{k}}^{\prime}\left(U^{\left(\lambda_{k}\right)}\right)=0, \quad I_{\lambda_{k}}\left(U^{\left(\lambda_{k}\right)}\right) \leq \sup _{\bar{Q}} I
$$

is the direct consequence of Lemma 6. To prove the boundedness of $\left\{U^{\left(\lambda_{k}\right)}\right\}$, we argue as follows.

By (A4) and the definition of $I_{\lambda_{k}}$ and $I_{\lambda_{k}}^{\prime}$, we have

$$
\begin{aligned}
\sup _{\bar{Q}} I & \geq I_{\lambda_{k}}\left(U^{\left(\lambda_{k}\right)}\right)=\lambda_{k} \sum_{n \in Z} \gamma_{n}\left(\frac{1}{2}\left(\nabla H\left(n, U_{n}^{\left(\lambda_{k}\right)}\right), U_{n}^{\left(\lambda_{k}\right)}\right)-H\left(n, U_{n}^{\left(\lambda_{k}\right)}\right)\right) \\
& \geq c \lambda_{k} \sum_{n \in Z} \gamma_{n}\left|U_{n}^{\left(\lambda_{k}\right)}\right|^{q} .
\end{aligned}
$$

By (A2) and the Hölder inequality, we obtain

$$
\begin{aligned}
\left\|U^{\left(\lambda_{k}\right)+}\right\|^{2}= & \lambda_{k} \sum_{n \in Z} \gamma_{n}\left(\nabla H\left(n, U_{n}^{\left(\lambda_{k}\right)}\right), U_{n}^{\left(\lambda_{k}\right)+}\right) \\
\leq & c \sum_{n \in Z} \gamma_{n}\left(\left|U_{n}^{\left(\lambda_{k}\right)+}\right|+\left|U_{n}^{\left(\lambda_{k}\right)}\right|^{p-1}\left|U_{n}^{\left(\lambda_{k}\right)+}\right|\right) \\
\leq & c\left(\sum_{n \in Z} \gamma_{n}^{2}\right)^{1 / 2}\left(\sum_{n \in Z}\left|U_{n}^{\left(\lambda_{k}\right)+}\right|^{2}\right)^{1 / 2} \\
& +c\left(\sum_{n \in Z} \gamma_{n}^{\frac{q}{p-1}}\left|U_{n}^{\left(\lambda_{k}\right)}\right|^{q}\right)^{(p-1) / q}\left(\sum_{n \in Z} \mid U_{n}^{\left.\left(\lambda_{k}\right)+\mid \frac{q}{q-p+1}\right)^{(q-p+1) / q}} .\right.
\end{aligned}
$$


Since $q /(p-1) \geq 1, q /(q-p+1) \geq 2$, and considering (A5) and (24), we obtain

$$
\left\|U^{\left(\lambda_{k}\right)+}\right\|^{2} \leq c\left\|U^{\left(\lambda_{k}\right)+}\right\|
$$

Therefore, $\left\|U^{\left(\lambda_{k}\right)+}\right\| \leq c$. Since $I_{\lambda_{k}}\left(U^{\left(\lambda_{k}\right)}\right) \geq 0$, we know $\left\|U^{\left(\lambda_{k}\right)-}\right\| \leq\left\|U^{\left(\lambda_{k}\right)+}\right\|$. The proof is completed.

Theorem 8 If assumptions (S0) and (A1)-(A5) are satisfied, then (5) (or (4)) has at least one nontrivial homoclinic solution.

Proof Let $\left\{U^{\left(\lambda_{k}\right)}\right\}$ be the sequence obtained in Lemma 7. Noting that $\left\{U^{\left(\lambda_{k}\right)}\right\}$ is bounded, and considering

$$
\left\langle I^{\prime}\left(U^{\left(\lambda_{k}\right)}\right), V\right\rangle=\left\langle I_{\lambda_{k}}^{\prime}\left(U^{\left(\lambda_{k}\right)}\right), V\right\rangle+\left(\lambda_{k}-1\right)\left(\left\langle U^{\left(\lambda_{k}\right)-}, V^{-}\right\rangle+\sum_{n \in Z} \gamma_{n}\left(\nabla H\left(n, U_{n}^{\left(\lambda_{k}\right)}\right), V\right)\right)
$$

for any $V \in l^{2}$ and

$$
I\left(U^{\left(\lambda_{k}\right)}\right)=I_{\lambda_{k}}\left(U^{\left(\lambda_{k}\right)}\right)+\left(\lambda_{k}-1\right)\left(\frac{1}{2}\left\|U^{\left(\lambda_{k}\right)-}\right\|^{2}+\sum_{n \in Z} \gamma_{n} H\left(n, U_{n}^{\left(\lambda_{k}\right)}\right)\right)
$$

we obtain

$$
\lim _{k \rightarrow \infty} I^{\prime}\left(U^{\left(\lambda_{k}\right)}\right)=0, \quad \lim _{k \rightarrow \infty} I\left(U^{\left(\lambda_{k}\right)}\right) \leq \sup _{\bar{Q}} I
$$

Since $\left\{U^{\left(\lambda_{k}\right)}\right\}$ is bounded, up to a sequence, we assume that $U^{\left(\lambda_{k}\right)} \rightarrow U$ in $l^{2}$. We claim that there exists a constant $\theta>0$ such that

$$
\lim _{k \rightarrow \infty}\left\|U^{\left(\lambda_{k}\right)}\right\| \geq \theta
$$

Indeed, if not, then $\lim _{k \rightarrow \infty}\left\|U^{\left(\lambda_{k}\right)}\right\|=0$. Since $I_{\lambda_{k}}^{\prime}\left(U^{\left(\lambda_{k}\right)}\right)=0$, and by (12), (17), and the Hölder inequality, we have

$$
\begin{aligned}
\left\|U^{\left(\lambda_{k}\right)+}\right\|^{2} & =\lambda_{k} \sum_{n \in Z} \gamma_{n}\left(\nabla H\left(n, U_{n}^{\left(\lambda_{k}\right)}\right), U_{n}^{\left(\lambda_{k}\right)+}\right) \\
& \leq c \sum_{n \in Z} \gamma_{n}\left(\epsilon\left|U_{n}^{\left(\lambda_{k}\right)}\right|+c_{\epsilon}\left|U_{n}^{\left(\lambda_{k}\right)}\right|^{p-1}\right)\left|U_{n}^{\left(\lambda_{k}\right)+}\right| \\
& \leq c \epsilon\left\|U^{\left(\lambda_{k}\right)}\right\|_{l^{2}}\left\|U^{\left(\lambda_{k}\right)+}\right\|_{l^{2}}+c_{\epsilon}\left\|U^{\left(\lambda_{k}\right)}\right\|_{l^{2}}^{p-1}\left\|U^{\left(\lambda_{k}\right)+}\right\|_{l^{2}},
\end{aligned}
$$

where $\epsilon>0$ is an arbitrarily small number, independently with $k$, and $c>0$ is a constant independently with $\epsilon$ and $k$. Similarly, we have

$$
\left\|U^{\left(\lambda_{k}\right)-}\right\|^{2} \leq c \epsilon\left\|U^{\left(\lambda_{k}\right)}\right\|_{l^{2}}\left\|U^{\left(\lambda_{k}\right)-}\right\|_{l^{2}}+c_{\epsilon}\left\|U^{\left(\lambda_{k}\right)}\right\|_{l^{2}}^{p-1}\left\|U^{\left(\lambda_{k}\right)-}\right\|_{l^{2}}
$$

From (27) and (28), we have

$$
\left\|U^{\left(\lambda_{k}\right)}\right\|^{2} \leq 2 c \epsilon\left\|U^{\left(\lambda_{k}\right)}\right\|\left\|U^{\left(\lambda_{k}\right)}\right\|+2 c_{\epsilon}\left\|U^{\left(\lambda_{k}\right)}\right\|^{p-1}\left\|U^{\left(\lambda_{k}\right)}\right\| .
$$


Choosing $\epsilon$ small enough such that $2 c \epsilon \leq 1 / 2$, and noting that $\left\|U^{\left(\lambda_{k}\right)}\right\| \neq 0$, we obtain $\left\|U^{\left(\lambda_{k}\right)}\right\| \geq c$ for some constant $c>0$. That is a contradiction. So (26) holds. Considering $\left\|U^{\left(\lambda_{k}\right)+}\right\| \geq\left\|U^{\left(\lambda_{k}\right)-}\right\|$, we obtain

$$
\lim _{k \rightarrow \infty}\left\|U^{\left(\lambda_{k}\right)+}\right\| \geq \theta / 2
$$

As for the proof of Step 1 in Lemma 6, we can prove that $U^{\left(\lambda_{k}\right)+} \rightarrow U^{+}$in $l^{2}$. Thus $U \neq 0$. Since $I^{\prime}\left(U^{\left(\lambda_{k}\right)}\right) \rightarrow 0$, we have $I^{\prime}(U)=0$. The proof is completed.

Remark 9 For a general discrete system of the form

$$
L U_{n}=\gamma_{n} \nabla H\left(n, U_{n}\right), \quad n \in Z, U_{n} \in R^{m}
$$

where $L$ is a bounded, self-adjoint, second-order linear difference operator and $0 \notin \sigma(L)$, $\sigma(L) \cap(0, \infty) \neq \varnothing$ and $\sigma(L) \cap(-\infty, 0) \neq \varnothing$, our method is also valid.

Remark 10 Similarly, we can consider the existence of homoclinic periodic solutions for the discrete wave equation of the form

$$
\left\{\begin{array}{l}
\Delta^{2} u_{n-1}^{i}-\delta^{2} \nabla^{2} u_{n}^{i-1}+\alpha u_{n}^{i}-\gamma_{n} f\left(n, u_{n}^{i}\right)=0, \quad i, n \in Z \\
\lim _{i \rightarrow \infty} u_{n}^{|i|}=0, \quad n \in Z
\end{array}\right.
$$

For example, let $T$ be a positive integer. It is well known that the eigenvalue problem of the form

$$
\left\{\begin{array}{l}
-\Delta^{2} u_{n-1}=\lambda u_{n}, \quad n \in[1, T], \\
u_{0}=u_{T}, \quad u_{1}=u_{T+1},
\end{array}\right.
$$

has the eigenvalues

$$
\lambda_{k}=4 \sin ^{2} \frac{k \pi}{T}, \quad k \in[1, T]
$$

In this case, the spectrum of $L^{\prime}$ is $\sigma\left(L^{\prime}\right)=\bigcup_{k=1}^{T}\left\{\delta^{2}[0,4]+\alpha-\lambda_{k}\right\}$. Thus, similar results can be obtained. They will be omitted.

Remark 11 Recently, the authors in [19] considered the existence of periodic and subharmonic solutions for the nonlinear difference equations with $p$-Laplace operator by using the saddle point theorem. On the other hand, when $\delta=1$, the linear part is nonnegative definite. In [20], they discussed a similar problem for a $2 n$ th-order nonlinear difference equation.

Remark 12 It is well known that the difference equations on time scales (see [21]) and the fractional finite difference equations (see [22] and [23] and the listed references) have been extensively investigated. Clearly, we can also consider similar problems, however, the matrix and vector methods cannot be used. 
Competing interests

The authors declare that they have no competing interests.

Authors' contributions

The authors contributed equally to this paper. All authors read and approved the final manuscript.

\section{Acknowledgements}

The authors would like to thank the anonymous referees for their comments and suggestions on the manuscript. This work was supported by the National Natural Science Foundation of China (No. 11371277).

\section{Received: 15 June 2015 Accepted: 11 November 2015 Published online: 24 November 2015}

\section{References}

1. Palmer, KJ: Shadowing in Dynamical Systems: Theory and Applications, vol. 501. Springer, Berlin (2000)

2. Šil'nikov, LP: Existence of a countable set of periodic motions in a neighborhood of a homoclinic curve. Dokl. Akad. Nauk SSSR 172, 298-301 (1967)

3. Smale, S: Differentiable dynamical systems. Bull. Am. Math. Soc. 73, 747-817 (1967)

4. Deng, $X Q$, Cheng, G: Homoclinic orbits for second order discrete Hamiltonian systems with potential changing sign. Acta Appl. Math. 103, 301-314 (2008)

5. Lin, GH, Zhou, Z: Homoclinic solutions of a class of nonperiodic discrete nonlinear systems in infinite higher dimensional lattices. Abstr. Appl. Anal. 2014, Article ID 436529 (2014)

6. Kuang, JH, Guo, ZM: Homoclinic solutions of a class of periodic difference equations with asymptotically linear nonlinearities. Nonlinear Anal. 89, 208-218 (2013)

7. Ma, M, Guo, ZM: Homoclinic orbits and subharmonics for nonlinear second order difference equations. Nonlinear Anal. 67, 1737-1745 (2007)

8. Zhang, X: Multibump solutions of a class of second-order discrete Hamiltonian systems. Appl. Math. Comput. 236, 129-149 (2014)

9. Zhou, Z, Yu, JS, Chen, Y: Homoclinic solutions in periodic difference equations with saturable nonlinearity. Sci. China Math. 54, 83-93 (2011)

10. Ma, M, Guo, ZM: Homoclinic orbits for second order self-adjoint difference equations. J. Math. Anal. Appl. 323(1), 513-521 (2006)

11. Chen, GW, Ma, SW: Discrete nonlinear Schrödinger equations with superlinear nonlinearities. Appl. Math. Comput. 218, 5496-5507 (2012)

12. Agarwal, RP: Difference Equations and Inequalities: Theory, Methods, and Applications. CRC Press, Boca Raton (2000)

13. Cheng, SS: Partial Difference Equations. Taylor \& Francis, London (2003)

14. Bates, PW, Zhang, C: Traveling pulses for the Klein-Gordon equation on a lattice or continuum with long-range interaction. Discrete Contin. Dyn. Syst. 16(1), 235-252 (2006)

15. Ragazzo, CG: Chaos and integrability in a nonlinear wave equation. J. Dyn. Differ. Equ. 6(1), 1-18 (1994)

16. Schechter, M, Zou, W: Weak linking theorems and Schrödinger equations with critical Sobolev exponent. ESAIM Control Optim. Calc. Var. 9, 601-619 (2003)

17. Teschl, G: Jacobi Operators and Completely Integrable Nonlinear Lattices, vol. 72. Am. Math. Soc., Providence (2000)

18. Stuart, CA: Bifurcation into spectral gaps. Bull. Belg. Math. Soc. Simon Stevin suppl., 59 pp. (1995)

19. Liu, X, Zhang, YB, Shi, HP: Existence of periodic solutions for a class of nonlinear difference equations. Qual. Theory Dyn. Syst. 14, 51-69 (2015)

20. Liu, X, Zhang, YB, Shi, HP: Periodic and subharmonic solutions for a 2 nth-order nonlinear difference equation. Hacet. J. Math. Stat. 44(2), 351-362 (2015)

21. Bohner, M, Peterson, A: Dynamic Equations on Time Scales: An Introduction with Applications. Birkhäuser, Basel (2001)

22. Agarwal, RP, Baleanu, D, Rezapour, S, Salehi, S: The existence of solutions for some fractional finite difference equations via sum boundary conditions. Adv. Differ. Equ. 2014, Article ID 282 (2014)

23. Wu, GC, Baleanu, D, Deng, ZG, Zeng, SD: Lattice fractional diffusion equation in terms of a Riesz-Caputo difference. Physica A 438, 335-339 (2015)

\section{Submit your manuscript to a SpringerOpen ${ }^{\circ}$ journal and benefit from:}

- Convenient online submission

Rigorous peer review

- Immediate publication on acceptance

- Open access: articles freely available online

- High visibility within the field

- Retaining the copyright to your article 